\title{
Quantifying Oxygen Vacancies in Fuel-Cells Materials using Atomic EELS Analysis
}

\author{
P. Longo ${ }^{1}$, M.F. Chisholm ${ }^{2}$, M. Varela ${ }^{2}$, A.R. Lupini ${ }^{2}$ and R.D. Twesten ${ }^{1}$ \\ ${ }^{1}$ Gatan Inc. 5794 W Las Positas Blvd, Pleasanton, CA, 94588, USA \\ ${ }^{2}$ Materials Science and Technology Division, Oak Ridge National Laboratory, Oak Ridge, \\ Tennessee 37831, USA
}

Fuel cells are promising systems for efficient energy conversion technology. Electrical energy is produced through a chemical reaction between oxygen and a fuel (typically $\mathrm{H}$ ). In solid oxide fuel cells, $\mathrm{O}$ ions move through a solid electrolyte via $\mathrm{O}$ vacancies hopping to react with the fuel. The density and distribution of $\mathrm{O}$ vacancies in electrolyte influence the performance of the overall device. Quantifying these O vacancies, down to the unit cell level, provide insight into the physical properties and chemical interactions that occur across different regions in fuel cell materials. This insight would help overcome performance barriers such as limited lifespan and low temperature operation that have prevented the widespread commercial use of fuel cells as energy converters.

Here we present an EELS based approach for direct quantification of oxygen vacancy concentration using electron energy-loss spectroscopy and a model based analysis approach [1]. For this study, a specimen of $\mathrm{SrTiO}_{3} / \mathrm{SrCoO}_{\mathrm{x}}(\mathrm{STO}) /(\mathrm{SCO})$ was analyzed. O vacancies can be easily observed in the $\mathrm{SCO}$ area as alternating dark contrast in the Sr-O atomic planes as shown in the analog ADF STEM image in Figure 1a. The distance between these planes varies from $0.32 \mathrm{~nm}$ and $0.38 \mathrm{~nm}$ where the oxygen vacancies are present. A similar spacing variation has been previously reported for LSCO thin films [2], where it was found to be the result of structural relaxation. Here elemental and compositional analysis was carried out using atomic level EELS analysis. Data were acquired using a Nion UltraSTEM operating at 200kV equipped with a cold-FEG and a Gatan Enfinium ER EELS spectrometer. For the results shown in this abstract, EELS spectra were acquired in single range mode using an exposure time as low as $8 \mathrm{~ms}$ per spectrum in order to limit the electron beam damage. O, Sr and Co EELS intensity maps are shown in Figures 1b-d. The effects of the presence of the structural relaxation typical of $\mathrm{O}$ vacancies can be observed in the Sr elemental map in Figure $1 \mathrm{c}$ as alternating dark contrast. However, $\mathrm{O}$ vacancies can be directly observed in the $\mathrm{O}$ elemental map in Figure 1b as alternating dark and bright stripes and can be quantified using a model based approach [1]. This is possible because the edges from $\mathrm{Sr} \mathrm{L}_{2,3}$ at $1940 \mathrm{eV}, \mathrm{Co} \mathrm{L}_{2,3}$ at $779 \mathrm{eV}$ and O K at $532 \mathrm{eV}$, in the SCO area, can be all recorded in the same EELS spectrum given the high-dynamic range available in the latest generation of EELS spectrometers as shown in Figure 2. The $\mathrm{O}$ concentration varies from $54 \%$ to $52 \%$, the latter in the area where the $\mathrm{O}$ vacancies are observed. Consistency of these results was also verified using a more conventional approach with a standard material containing the same elements $\mathrm{Co}, \mathrm{Sr}$ and $\mathrm{O}$. The stripes in the $\mathrm{O}$ elemental map fade during the acquisition; this is due to electron beam damage. In addition the image contrast at the interface seems to be affected by misfit strain.

These preliminary results demonstrate the consistency and the accuracy of the EELS quantification routine. Other materials containing $\mathrm{O}$ vacancies are currently under investigation using the same approach, and the results will be also presented. These materials range from $\mathrm{GdCeOx}, \mathrm{LaSrCoOx}$ to LaSrMnOx. They are being investigated using means of acquisition in DualEELS ${ }^{\mathrm{TM}}$ mode where the low- and high-loss regions of the EELS spectrum are acquired nearly simultaneously under the same 
experimental conditions [3]. Having the low-loss contribution allows correction for the effects of multiple scattering that affects the shape of the edges and allows absolute quantification of the $\mathrm{O}$ content. Acquiring data in DualEELS ${ }^{\mathrm{TM}}$ mode opens up new avenues for the characterization of such materials and will allow crucial quantitative compositional information to be obtained.

References:

[1] P.J. Thomas et al., Microscopy and Microanalysis, 18, 968, 2012

[2] Y-M Kim et al., Nature Materials, 11, 888-894, 2012

[3] A.J. Gubbens et al., Ultramicroscopy, 110, 962-970, 2010

[4] Research supported by the U.S. Department of Energy, Basic Energy Sciences and Engineering Division (MFC, MV, ARL)
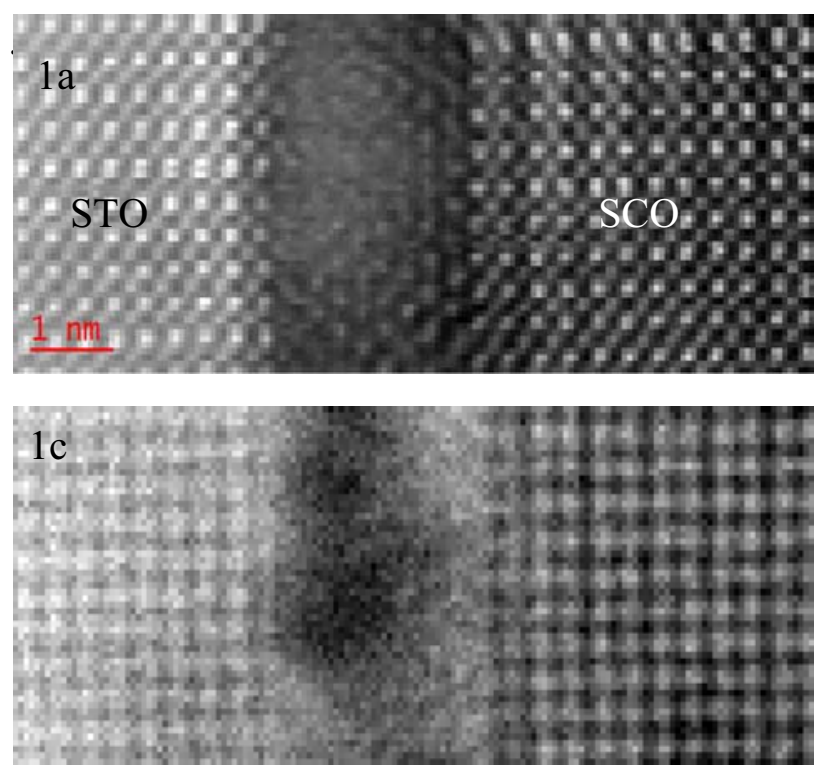
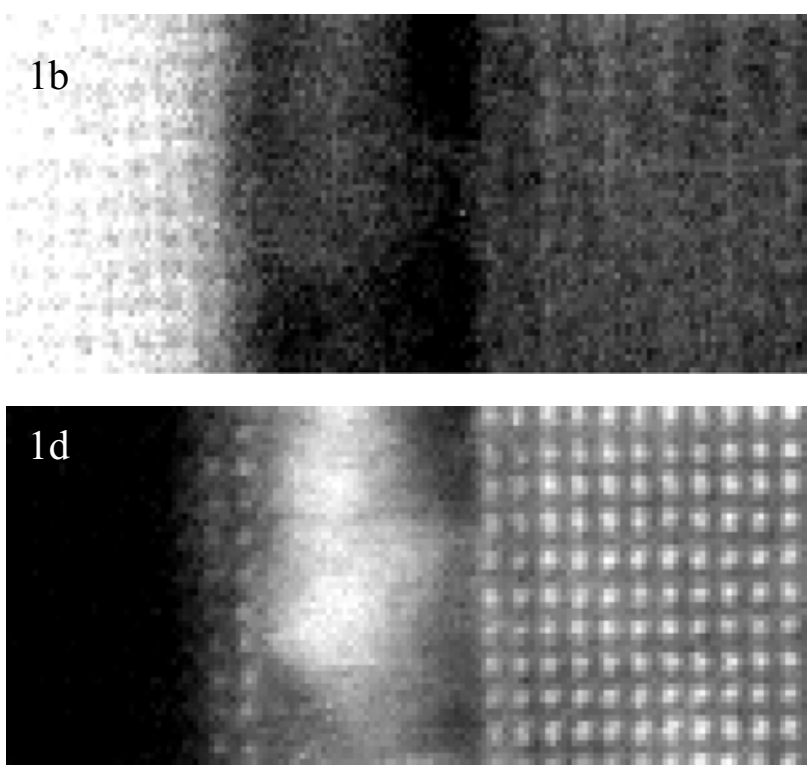

Figures 1. a): ADF STEM image taken simultaneously during the acquisition of the spectrum imaging. The alternating dark contrast shows where the $\mathrm{O}$ vacancies are expected; $\mathrm{b}$ ) $\mathrm{O}$ elemental map showing the presence of $\mathrm{O}$ vacancies in the dark stripes; c) Sr and d) Co elemental maps.

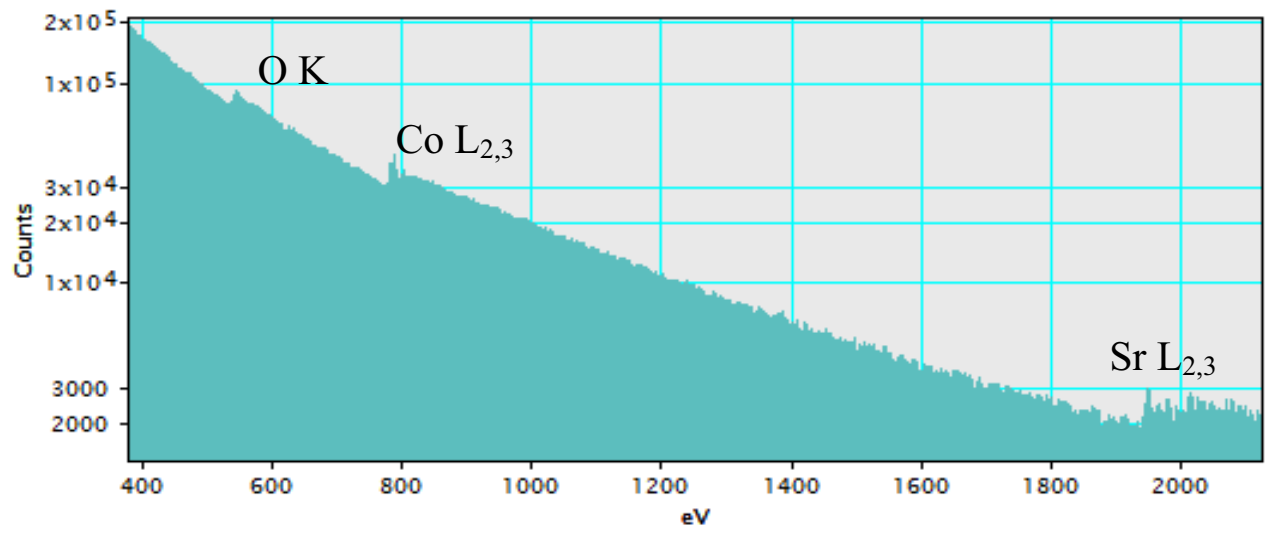

Figure 2. EELS spectrum in log-view extracted from the SCO region extending from $400 \mathrm{eV}$ to $2400 \mathrm{eV}$. All the edges from $\mathrm{Sr} \mathrm{L}_{2,3}$ at $1940 \mathrm{eV}, \mathrm{Co} \mathrm{L}_{2,3}$ at $779 \mathrm{eV}$ and $\mathrm{O} \mathrm{K}$ at $532 \mathrm{eV}$ recorded in the same spectrum, allowing the relative composition to be accurately determined. 